Dominik Mann, Sean-Pascal Kuttner, Julius Georgi Wesirow, Lukas Bachmann, Anna Marie Körner, Yannis Grabbe, Laura Rothe, Lisa Schueler, Julia Lang, Michaela Fuchs

\title{
Applaus allein reicht nicht - wie können Pflegeberufe attraktiver werden?
}

\begin{abstract}
Die Sicherstellung von genügend Altenpfleger:innen stellt eine sehr große Herausforderung dar, die sich in den kommenden Jahren noch verstärken wird. Sowohl die derzeitigen Pflegekräfte in ihren Jobs zu halten als auch mehr Menschen für diesen Beruf zu gewinnen, ist daher unabdingbar für die Gewährleistung der bedarfsgerechten pflegerischen Versorgung. Einen Baustein hierfür stellt das Projekt „CarEducation“ dar, für das die Schüler:innen des Landschulheims Grovesmühle beim diesjährigen YES!-Wettbewerb mit dem „Best Scientific Analysis Award“ ausgezeichnet wurden. Es soll Schüler:innen sachlich über den Beruf informieren, Vorurteile nehmen und die Begeisterung für Pflege im Allgemeinen steigern.
\end{abstract}

\begin{abstract}
Der demografische Wandel in Deutschland äußert sich in der Schrumpfung und der Alterung der Bevölkerung. In Zukunft werden damit nicht nur weniger Menschen in Deutschland leben, sondern auch die Zahl der Älteren wird deutlich steigen. Diese Entwicklungen zeigen sich besonders anschaulich anhand der stark besetzten Jahrgänge von 1955 bis 1970, die zur sogenannten Babyboom-Generation gehören (Statistisches Bundesamt, 2019). Sie waren 2019 zwischen 49 und 64 Jahre alt und stellten die größte Altersgruppe dar (vgl. Abbildung 1). In den kommenden Jahren werden sie sukzessive aus dem Erwerbsalter ausscheiden, was - zusammen mit dem Nachrücken vergleichsweise gering besetzter Jahrgänge - die Zahl der für das Arbeitsangebot relevanten Einwohner:innen im erwerbsfähigen Alter zwischen 15 und 65 Jahren deutlich reduzieren wird.

(c) Der/die Autor:in 2021. Open Access: Dieser Artikel wird unter der Creative Commons Namensnennung 4.0 International Lizenz veröffentlicht (creativecommons.org/licenses/by/4.0/deed.de).

Open Access wird durch die ZBW - Leibniz-Informationszentrum Wirtschaft gefördert.
\end{abstract}

Dominik Mann, Sean-Pascal Kuttner, Julius Georgi Wesirow, Lukas Bachmann, Anna Marie Körner, Yannis Grabbe, Laura Rothe und Lisa Schueler sind Schüler:innen des Landschulheims Grovesmühle.

Dr. Julia Lang und Dr. Michaela Fuchs sind wissenschaftliche Mitarbeiterinnen am Institut für Arbeitsmarkt- und Berufsforschung (IAB), Nürnberg.
Auf den Bereich der Altenpflege wirkt sich die demografische Entwicklung in doppelter Weise gravierend aus. Zum einen wird die Nachfrage nach professionellen Pflegedienstleistungen steigen, da aufgrund der zunehmenden Zahl an hochbetagten Menschen mit einer Zunahme der Pflegebedürftigen gerechnet werden muss (Fuchs, 2019; Schwinger, Klauber und Tsiasioti, 2020). Zum anderen steht zu befürchten, dass das Angebot an Pflegedienstleistungen sinken wird, da im Zuge des Rückgangs der Einwohner:innen im erwerbsfähigen Alter auch das Angebot an Arbeitskräften sinken wird (Fuchs, Söhnlein und Weber, 2017).

Der Arbeitsmarktsituation in der Pflege kommt damit eine besondere Bedeutung zu. Allerdings ist sie schon heute als kritisch einzustufen, denn in den Berufen der Altenpflege existiert ein ausgeprägter Fachkräfteengpass (DIP, 2018). 2020 standen 100 bei der Bundesagentur für Arbeit gemeldeten Stellen für Altenpflegefachkräfte lediglich 26 Arbeitslose in diesem Bereich gegenüber (Statistik der BA, 2021). Als wichtige Ursachen für den Engpass gelten niedrige Gehälter (Carstensen, Seibert und Wiethölter, 2021) und schlechte Arbeitsbedingungen, die sich in Zeitdruck, körperlicher Schwerarbeit, hohen emotionalen Belastungen oder unregelmäßigen Arbeitszeiten widerspiegeln (DGB-Index Gute Arbeit, 2018). In der Folge verlassen viele Pflegearbeitskräfte ihren Beruf und orientieren sich beruflich neu (Fuchs und Weyh, 2018).

Angesichts dieser Entwicklungen gewinnt die Frage zunehmend an Bedeutung, inwiefern die adäquate Versorgung der Pflegebedürftigen auch in Zukunft gewährleistet werden kann. Neben einem vorzeitigen Ausstieg aus dem Beruf wird befürchtet, dass das Ausbildungsinteresse an 
Abbildung 1

Altersaufbau der Bevölkerung in Deutschland, 2019

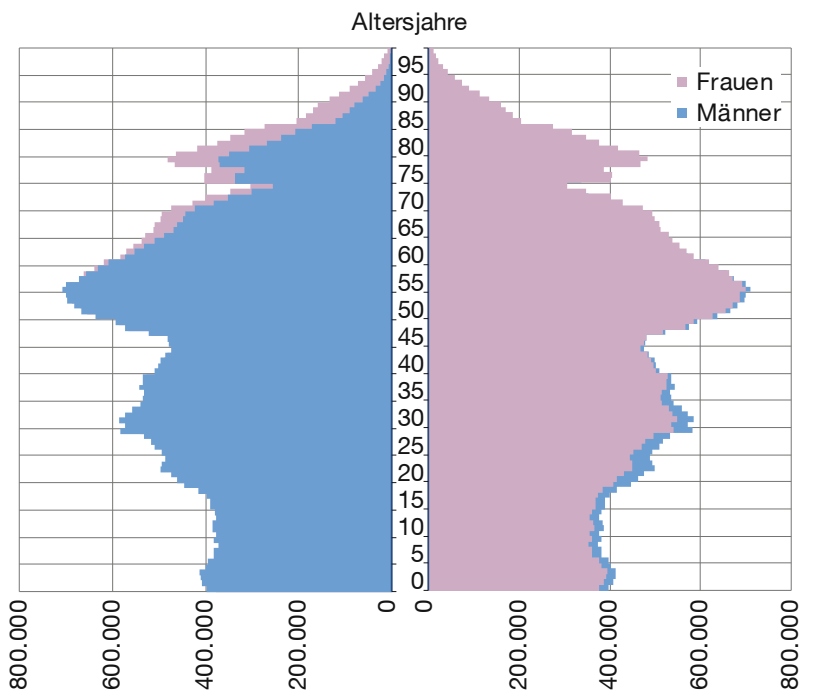

Anmerkung: Bevölkerung zum Stichtag 31.12.2019.

Quelle: Statistisches Bundesamt; eigene Darstellung.

den Pflegeberufen sinken wird (DIP, 2018). Maßnahmen, die darauf abzielen, mehr Menschen für diesen Beruf zu begeistern, stellen daher eine zentrale Stellschraube dar, um die bedarfsgerechte pflegerische Versorgung weiterhin sicherzustellen. Das Projekt des Landschulheims Grovesmühle ist hierfür ein sehr gelungenes Beispiel.

\section{Das Projekt: CarEducation}

Wir haben uns in unserem Projekt darauf konzentriert, den Pflegeberuf auch bei jüngeren Schüler:innen realistisch darzustellen. Wir wollen nichts beschönigen, aber wir wollen auch mit bestehenden Vorurteilen aufräumen, um den Schüler:innen ein objektives Bild zu ermöglichen. Unsere Idee für das Projekt war zu informieren und aufzuklären. Um das Projekt auch leicht an anderen Schulen nutzbar zu machen und es Schüler:innen zu ermöglichen, überall und jederzeit Zugang zu Informationen zum Pflegeberuf zu erhalten, entwickelten wir CarEducation. Es besteht aus drei Bausteinen und stellt einen neuen Ansatz dar, ein Projekt zu gestalten. Neben einem Projekttag umfasst CarEducation ein umfangreiches Portfolio, das sich mit der Pflege und ihren verschiedenen Teilgebieten auseinandersetzt. Darüber hinaus beinhaltet es einen in das Portfolio integrierten Lehrpfad.

Wir konzipierten unser Projekt als Projekttag, doch das bereitgestellte Material ist so umfangreich, dass damit auch eine Projektwoche gestaltet werden könnte. Was unser Projekt besonders macht, sind das Portfolio und der Lehrpfad. Wir haben dort verschiedene Themenbe- reiche wie Zukunft, Bildung, Geschichte und Image des Pflegeberufs eingegliedert.

Zudem haben wir einen universell für alle Kategorien geltenden Einstieg vorbereitet, der den Schüler:innen als Arbeitsgrundlage dienen soll. Für die jeweiligen Themenbereiche haben wir verschiedene Aufgaben angefertigt, sogenannte Basis- und Wahlaufgaben. Die Basisaufgaben sollen ein grundlegendes Verständnis zum Themenkomplex geben, während die Wahlaufgaben weiterführend in detailliertere Bereiche der Pflege vordringen. Im Themenbereich Zukunft etwa sollen sich Schüler:innen mit dem demografischen Wandel, mit der Entlohnung von Pflegekräften oder mit Pflegerobotern beschäftigen. Im Themenbereich Bildung geht es um die Ausbildungsmöglichkeiten, das neue Pflegestudium oder Aufstiegschancen im Pflegeberuf. Im Bereich Geschichte wiederum geht es um verschiedene Zeitepochen und um die jeweilige Rolle der Pflege, im Bereich Image um Vorurteile gegenüber Pflegekräften sowie um Arbeitsbedingungen.

Im Portfolio sind alle Facetten des Pflegeberufs, von theoretischen Überlegungen bis hin zur praktischen Umsetzung, untergebracht. Damit das Projekt für die Schüler:innen nicht einfach nur „ein weiterer Tag ohne Unterricht" ist, haben wir viele verschiedene Methoden für die jeweiligen Aufgaben eingebracht, sodass der Projekttag abwechslungsreich vonstatten gehen kann. Es gibt Einzel-, Partner-, sowie Gruppenarbeit, Aufgaben auf Grundlage von Grafiken oder Texten, aber auch Aufgaben mit integrierter Diskussion, oder z. B. eine Aufgabe zur Erstellung eines Mini-Theaterstücks.

Um außerdem zu ermöglichen, dass das Projekt in verschiedenen Jahrgängen stattfinden kann, haben wir jeder Aufgabe einen „Sternchen-Wert“ zugeschrieben. Ein Stern bedeutet, dass die Aufgabe leicht/wenig theoretisch ist, fünf Sterne bedeuten, dass die Aufgabe eher aufwendig ist. So können die Lehrkräfte auch anhand der Situation entscheiden, welche Aufgaben die Klassen noch mit Interesse durchführen könnten. Da der Projekttag grundsätzlich ohne Vorbereitung erfolgen sollte, haben wir vor den Aufgaben die benötigten Materialien beschrieben.

Der dritte Teil unseres Projekts ist der integrierte Lehrpfad. Die Idee ist, dass eine Lehrkraft damit auch kurzfristig einen Projekttag planen könnte und kein detailliertes Fachwissen benötigt. Im integrierten Lehrpfad finden sich alle Lösungen zu den gestellten Aufgaben. Sie beinhalten teilweise noch weiterführende Informationen sowie Anregungen, mit denen die Lehrkraft die Schüler:innen zum Nachdenken oder zum weiteren Recherchieren anregen kann. Alle Informationen sind so aufbereitet, dass die Lehrkraft sich nicht individuell vorzubereiten braucht. Auch Hinter- 
grundwissen ist nicht zwingend notwendig. Lehrer:innen erhalten dennoch Gelegenheit, ihre eigenen pädagogischen Methoden einzubauen. Die Aufgabenstellungen können zudem auch flexibel erweitert werden, etwa durch spontane Diskussions- oder Expert:innenrunden.

\section{Erfahrungen und weitere Entwicklung}

Wir haben das Projekt bereits an unserer Schule durchgeführt und sind auf eine sehr große, positive Resonanz gestoßen. Im ersten Durchlauf haben sich die Schüler:innen besonders mit den Teilgebieten Zukunft und Image beschäftigt. Um die Wirkung und den Nutzen des Projekts zu prüfen, haben wir eine Umfrage vor und nach dem Projekt durchgeführt: Während viele Schüler:innen dem Projekt anfangs skeptisch gegenüberstanden, waren sie im Nachgang sehr positiv sowohl gegenüber dem Projekt als auch gegenüber dem Beruf eingestellt $(90 \%$ der Befragten fanden den Projekttag eher oder sehr erfolgreich). In einer Diskussionsrunde haben wir festgestellt, wie hautnah Pflege bereits von vielen erlebt wird. Viele Schüler:innen sagten, dass sie in ihrer Verwandtschaft und im Freundeskreis sowohl Menschen haben, die in der Pflege tätig sind, als auch Menschen, die gepflegt werden. Das hat uns die Relevanz und die Allgegenwärtigkeit des Themas noch einmal vor Augen geführt.

Nach diesem erfolgreichen Durchlauf wollen wir unser Projekt auch an anderen Schulen durchführen. Die Neue Schule Wolfsburg ist seit dem Regionalfinale des YES!Wettbewerbs an unserem Projekt interessiert. In Zukunft werden wir das Projekt auch dort durchführen lassen, so können wir den integrierten Lehrpfad weiter erproben. Zudem können wir uns vorstellen, unser Projekt in Schulen in ganz Deutschland durchzuführen. Zuerst in unserem Landkreis, da wir dort Kontakte haben, dann möglicherweise in Schulen in einem anderen Bundesland und schließlich sogar bundesweit. Zudem wird unser Projekt in ein Jahrgangsprojekt umgewandelt, sodass jede 11. Klasse unserer Schule in Zukunft dieses Projekt weiterentwickeln kann.

Darüber hinaus haben wir noch weitere Ziele. Aufgrund unserer positiven Zusammenarbeit mit der Gemeinnützigen Gesellschaft für Sozialeinrichtungen (GSW) in Wer- nigerode setzen wir z.B. auf weitere Kooperationen mit Pflegeinstitutionen. Zudem sollen unser Projekt und die damit verbundene Thematik mithilfe der Medien populärer gemacht werden. Da wir uns bereits mit dem Bundesministerium für Wirtschaft und Energie in Verbindung gesetzt haben, hoffen wir auf weitere Unterstützung.

\section{Fazit}

Abschließend können wir sagen, dass CarEducation in vielerlei Hinsichten ein voller Erfolg ist. Da die Pflege ein wichtiges Thema in unserer Gesellschaft ist und wir spätestens im Alter wahrscheinlich alle auf Pflegekräfte angewiesen sein werden, war es uns sehr wichtig, dieses Projekt auszuarbeiten und die Thematik des Pflegemangels vielen Personen näher zu bringen. YES! hat uns die Möglichkeiten gegeben, auf dieses Thema aufmerksam zu machen. Die dortige Resonanz hat uns bestätigt, dass es auch vielen jüngeren Menschen wichtig ist, über das Thema Pflege aufgeklärt zu werden und dieses Tätigkeitsfeld bei ihrer Berufswahl zu berücksichtigen.

\section{Literatur}

Carstensen, J., H. Seibert und D. Wiethölter (2021), Entgelte von Pflegekräften 2020, Institut für Arbeitsmarkt- und Berufsforschung, Aktuelle Daten und Indikatoren.

DIP - Deutsches Institut für angewandte Pflegeforschung (2018), PflegeThermometer 2018

DGB-Index Gute Arbeit (2018), Arbeitsbedingungen in der Alten- und Krankenpflege - So beurteilen die Beschäftigten die Lage, Ergebnisse einer Sonderauswertung der Repräsentativumfragen.

Fuchs, J., D. Söhnlein und B. Weber (2017), Projektion des Erwerbspersonenpotenzials bis 2060: Arbeitskräfteangebot sinkt auch bei hoher Zuwanderung, IAB-Kurzbericht, 6.

Fuchs, M. und A. Weyh (2018), Der Pflegearbeitsmarkt in Thüringen: Eine Bestandsaufnahme, IAB-Regional. Berichte und Analysen aus dem Regionalen Forschungsnetz, IAB Sachsen-Anhalt-Thüringen, 3.

Fuchs, M. (2019), Der zukünftige Beschäftigungsbedarf in der ambulanten und stationären Pflege in Thüringen: Modellrechnungen auf Kreisebene bis zum Jahr 2035, IAB-Regional. Berichte und Analysen aus dem Regionalen Forschungsnetz, IAB Sachsen-Anhalt-Thüringen, 2.

Schwinger, A., J. Klauber und C. Tsiasioti (2020), Pflegepersonal heute und morgen, in K. Jacobs, A. Kuhlmey, S. Greß, J. Klauber und A. Schwinger (Hrsg.), Pflege-Report 2019, Springer.

BA - Statistik der Bundesagentur für Arbeit (2021), Berichte: Blickpunkt Arbeitsmarkt - Arbeitsmarktsituation im Pflegebereich.

Statistisches Bundesamt (2019), Bevölkerung im Wandel. Annahmen und Ergebnisse der 14. koordinierten Bevölkerungsvorausberechnung.

Title: Applause Alone Is Not Enough - How Can Nursing Professions Become More Attractive?

Abstract: Demographic change has increased the need for elderly care. Ensuring a sufficient number of workers in this field is a major challenge that will become even more severe in the coming years. To ensure that the provision of care is in line with demand, it is necessary to keep the current nursing staff in their jobs and attract more people to this profession. The "CarEducation" project, for which the students of the Landschulheim Grovesmühle were awarded the "Best Scientific Analysis Award" at this year's YES!, can contribute. The project is designed to provide students with information about the profession, to reduce prejudices and to increase enthusiasm for nursing in general. To this end, a portfolio is provided that teachers can use to design a project day on the topic of elderly care.

JEL Classification: J11, J22, J23 